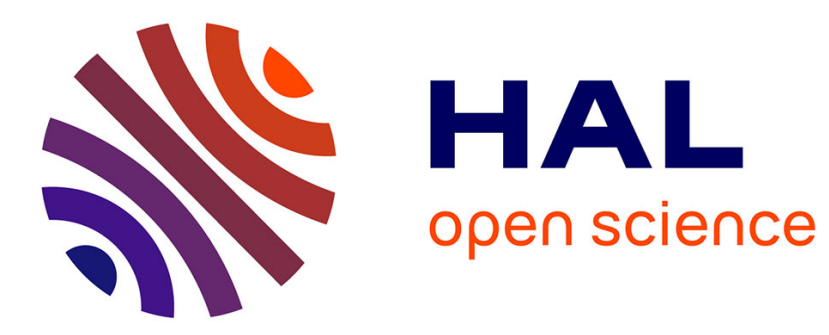

\title{
Les causes mineures du tribunal de Cuenca dans la deuxième moitié du XVIe siècle \\ Pascal Gandoulphe
}

\section{To cite this version:}

Pascal Gandoulphe. Les causes mineures du tribunal de Cuenca dans la deuxième moitié du XVIe siècle. Marie-Catherine Barbazza. L'inquisition espagnole et ses réformes au XVIe siècle, , pp.231-241, 2006, 9782367810935. hal-01527038

\section{HAL Id: hal-01527038 \\ https://hal.science/hal-01527038}

Submitted on 24 May 2017

HAL is a multi-disciplinary open access archive for the deposit and dissemination of scientific research documents, whether they are published or not. The documents may come from teaching and research institutions in France or abroad, or from public or private research centers.
L'archive ouverte pluridisciplinaire HAL, est destinée au dépôt et à la diffusion de documents scientifiques de niveau recherche, publiés ou non, émanant des établissements d'enseignement et de recherche français ou étrangers, des laboratoires publics ou privés. 


\section{9 boooks}
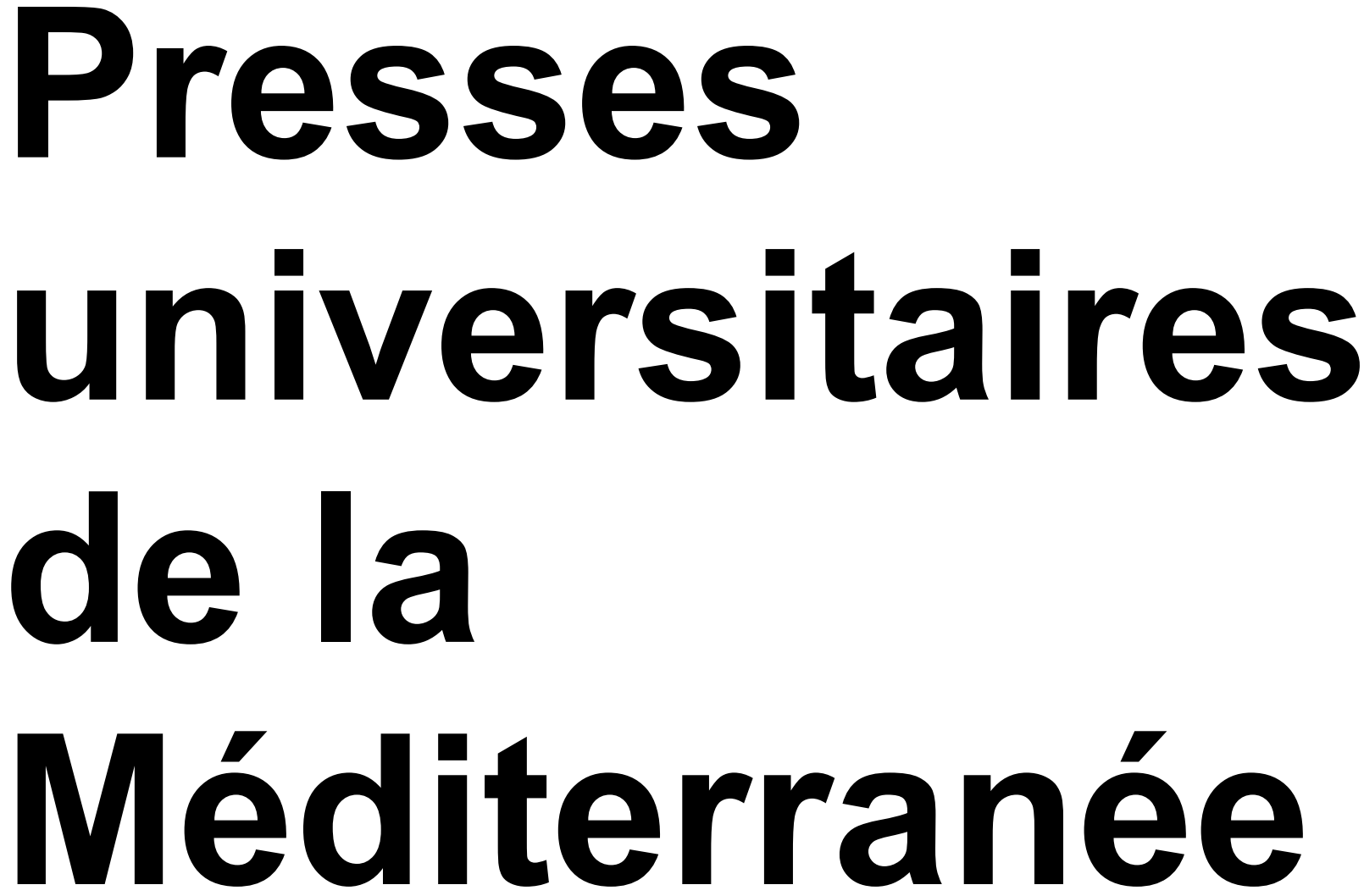

L'Inquisition espagnole et ses réformes au $X V l^{e}$ siècle | Marie-Catherine Barbazza

\section{Les causes} mineures du tribunal de Cuenca dans la deuxième 


\section{moitié du XVI ${ }^{e}$ \\ siècle}

Pascal Gandoulphe

p. 231-251

\section{Texte intégral}

L'on sait que pendant les cinquante premières années de son activité, l'Inquisition espagnole s'est employée presque exclusivement à poursuivre, condamner et faire exécuter des judéo-convers accusés de pratiquer en secret la religion de Moïse. À l'issue de ce premier temps de l'histoire de l'Inquisition espagnole que l'on peut assimiler à une vaste entreprise de « dé-sémitisation » de l'Espagne, les tribunaux péninsulaires ont élargi leur champ d'activité. Outre la répression qui s'abattit sur les nouveaux convertis de maures, les morisques, et sur les adeptes des formes nouvelles de la spiritualité chrétienne, l'attention prêtée aux petites erreurs en matière de doctrine est le fait saillant de la période qui s'ouvre autour des années 1530 : naguère marginale, cette part de l'activité du Saint-Office prit une importance croissante après la répression dont furent l'objet les cercles d'illuminés et d'érasmistes coupables de propager des idées et de prôner des attitudes que l'on en vint à assimiler aux thèses luthériennes.

2 Les affaires qui nous intéressent ici sont des causes dites mineures. Dans cette catégorie aux contours assez peu définis, l'historiographie a coutume de classer les affaires où sont en cause des opinions et des conduites extrêmement variées. Il s'agit tout d'abord de cet ensemble de délits de paroles et d'erreurs de jugement, que les inquisiteurs appelaient «propositions » dont l'éventail allait de la simple incongruité jusqu'à l'opinion pouvant être considérée comme hérétique, mais aussi d'un certain nombre de conduites considérées comme déviantes ou contraires à l'ordre religieux du monde (sorcellerie, 
bigamie, sollicitation, blasphème, outrage et impiété, sodomie et bestialité). Ces pratiques étaient déjà punies par les juridictions civile ou ecclésiastique depuis bien avant l'établissement de l'Inquisition espagnole.

Quelques recherches ont mis en lumière divers aspects quantitatifs et qualitatifs de cette répression massive affectant principalement, pour reprendre la formulation de Jean-Pierre Dedieu, des « individus sans relief particulier, qui se singularisent plus souvent par leur ignorance que par leur culture ${ }^{1}$. Mais, mis à part dans les travaux de Jean-Pierre Dedieu et de quelques autres ${ }^{2}$, cet aspect de l'activité inquisitoriale occupe d'ordinaire une place très secondaire, loin derrière les études consacrées aux féroces répressions exercées à l'encontre des judéo-convers, des morisques et, dans une moindre mesure, des élites lettrées s'inscrivant dans le vaste mouvement d'aspiration à la réforme religieuse. Il semblerait que cette facette-là de la répression inquisitoriale ne corresponde pas à l'image que l'on se fait de la terrible institution, parce qu'elle a pour cible un public indéterminé et proprement « vulgaire » et parce qu'elle fait preuve, dans ces affaires, d'une relative mansuétude : mis à part les sodomites dans la couronne d'Aragon, elle n'envoie jamais au bûcher ceux qu'elle condamne pour ces délits mineurs. En quelque sorte, cette inquisition-là déçoit.

4 Or, c'est pour ces raisons justement, parce qu'elle concerne la société espagnole tout entière, vieux et nouveaux chrétiens confondus, et que, pour légères que soient les peines qu'elle inflige à ses victimes, elle participe de l'imposition d'une nouvelle discipline religieuse et sociale, que cette inquisitionlà me semble particulièrement intéressante à étudier. Les objectifs qu'elle poursuit me semblent en effet radicalement différents de ceux qui avaient été les siens lors de la période antérieure : en tant qu'institution coercitive prenant part à l'entreprise de clarification et de diffusion des postulats religieux propres à l'Église romaine, mais aussi en tant qu'institution étroitement liée à un pouvoir monarchique de plus en plus hégémonique, l'Inquisition apparaît comme l'un des 
instruments de disciplinement religieux, social et politique à l'œuvre dans cette région de l'Europe catholique qu'est l'Espagne ${ }^{3}$.

Je me propose ici de rappeler dans un premier temps la nature de cette répression et les flux des principaux délits mineurs condamnés par le Saint-Office castillan dans la deuxième moitié du XVI ${ }^{\mathrm{e}}$ siècle. L'étude des sentences et des peines infligées retiendra ensuite mon attention, afin de mettre en lumière les stratégies suivies par ce tribunal dans cette entreprise de disciplinement religieux, social et politique. Enfin, l'examen de l'origine géographique des condamnés permettra de montrer les limites du contrôle exercé par le tribunal de Cuenca sur le territoire soumis à sa juridiction.

6 Pendant la seconde moitié du $\mathrm{XVI}^{\mathrm{e}}$ siècle, le tribunal de Cuenca consacra la plus grande part de son activité aux causes dites mineures : elles représentent plus des trois quarts des affaires traitées. Le processus avait commencé plus tôt, à la fin des années 1516-1520. Mais après un premier pic autour de 1525, suivi d'un ralentissement jusqu'à la fin des années 1550, le nombre de procès augmente d'une manière significative avant d'entamer une baisse, pendant la période 1586-90, qui préfigure la lente diminution de cette facette de l'activité des tribunaux inquisitoriaux jusqu'à leur disparition ${ }^{4}$.

7 Tout au long de la période concernée par cette étude, à l'exception des conduites de résistance et d'opposition au Saint-Office, seuls trois délits dépassent $5 \%$ des affaires traitées par le tribunal de Cuenca : il s'agit des « propositions » qui représentent, toutes natures confondues, un peu plus de la moitié (52\%) des causes jugées, du blasphème $(20,5 \%)$ et de la bigamie $(5 \%)^{5}$. Un quatrième délit retiendra notre attention : la sollicitation. Les prêtres sollicitants étaient coupables d'abuser de leurs pénitentes pendant la confession, parfois même, ce qui était plus grave, de subordonner l'absolution des péchés confessés à l'acceptation de pratiques sexuelles. Bien que moins représentés dans la totalité des causes jugées, le nombre de sollicitants (72, dont 53 entre 1580 et 1590 ) est 
loin d'être insignifiant compte tenu de la population spécifique concernée par ce délit.

8 D'après nos sources, deux de ces quatre délits semblent avoir fait l'objet d'une répression stable en valeurs relatives : il s'agit des délits de la parole. Les condamnations pour " propositions », quel qu'en soit le contenu, et celles pour blasphèmes ne présentent pas de variation significative au cours des quarante années étudiées.

9 En revanche, les condamnations pour bigamie sont plus fréquentes au début de la période, autour des années 1560 (91 des 130 cas entre 1556 et 1570) alors que l'on trouve la plus large part des prêtres sollicitants à la fin de la période (53 des 72 cas entre 1576 et 1590). Une telle variation traduit l'existence d'une politique visant à orienter la répression vers des cibles précises révélatrices des enjeux du temps. Pour ce qui est de la sollicitation, le point de départ de cette répression est facile à localiser dans le temps : c'est en 1561 que le pape Pie IV confia à l'Inquisition le soin de châtier les confesseurs indélicats. Quant à la bigamie, ce délit était considéré comme relevant d'une double juridiction, civile et religieuse, et l'Inquisition espagnole s'en empara sans toutefois exclure l'intervention de l'ordinaire ni celle de la justice civile.

10 Revenons aux délits de la parole, aux propositions notamment. La stabilité du flux masque en fait un recentrage sur une affirmation particulière, plus connue sous le nom de « simple fornication » dont la condamnation commence à la fin des années 1550 avant d'entrer officiellement dans le champ d'action de l'Inquisition par deux cartas acordadas dépêchées par le Conseil Suprême de l'Inquisition en 1573 et 1574. À la suite de quoi, pendant la décennie qui suivit, la répression de l'opinion selon laquelle la « simple fornication ${ }^{6}$ n'était pas un péché mortel, mais simplement véniel, devint la première source de procès du tribunal de Cuenca ${ }^{7}$.

11 L'objectif poursuivi par le Saint-Office en la matière, une véritable « campagne d'opinion » selon les termes de Jean-Pierre Dedieu, n'était autre que de rappeler à la population le caractère peccamineux de toute sexualité non 
matrimoniale, qu'il s'agisse d'une relation librement consentie par deux adultes libres de tout lien ou bien du commerce des prostituées.

La progression du nombre de procès est révélatrice ${ }^{8}$ de la répression croissante d'une opinion largement répandue.

Trois points de vue se dégagent des récits des condamnés pour ce délit. Dans la majorité des cas, il s'agissait d'une justification des relations charnelles avec des prostituées. Les mujeres del partido, ou de la mancebía étaient considérées par beaucoup comme de simples commerçantes. Ensuite, connaître, dans le sens biblique du terme, une prostituée ne pouvait être un péché mortel puisque la prostitution était encadrée par les autorités municipales qui percevaient une taxe sur l'activité des mancebías. Sans parler de la prostitution sauvage qui était largement répandue. Ainsi, une femme du nom de María García fut-elle condamnée à abjuer de levi en 1590 pour avoir dit que : "bien entendía ella que hera peccado tratar con ellas mas pues las consentía el Rey y el Papa que no era peccado mortal ${ }^{9}$. Un jeune homme incrédule connut le même sort pour s'être exclamé qu'il était impossible que : «Si una muger que estava en la putería tuviese que hazer con ochenta hombres, que ochenta pecados mortales avía de hazer ${ }^{10} »$.

14 Plus rarement, les condamnés revendiquaient une certaine liberté sexuelle et le pouvoir d'user de leur corps à leur guise. Un certain Juan de Ortega dut abjurer de levi en 1582 pour avoir affirmé qu'aucun péché n'était commis lorsque l'homme et la femme étaient simplement d'accord pour entretenir ensemble une relation sexuelle ${ }^{11}$. Un conte folklorique amusant, dont Jean Pierre Dedieu cite l'une des multiples variantes ${ }^{12}$, et que nous avons retrouvé à Cuenca, semble jouer le rôle de mythe justificateur de cette liberté sexuelle. Il met en scène Jésus-Christ et Saint Pierre : «Andando nuestro señor Jesuchristo y San Pedro por el mundo, San Pedro avía dado tras una tabernera y Nuestro Señor le avía dicho : "¿Qué haces Pedro ? »Y respondió : «Señor, jodo. »Y Jesuchristo le dixo : " iPues jode y vente ! $»^{13}$. L'on a même trouvé une variante où il est 
question de sainte Pétronile, fruit de cette éphémère liaison de saint Pierre avec l'aubergiste. Marco Pérez, qui racontait cette histoire en 1560, dut abjurer de levi et faire pénitence un dimanche, à l'église ${ }^{14}$.

15 Enfin, le troisième point de vue qu'exprimaient certains accusés était une défense de la sexualité pré-matrimoniale des futurs époux. La lecture de quelques procès pour ce délit semble montrer que, notamment dans les zones rurales, cette pratique n'était pas rare. Mais cette question avait fait l'objet de nombreux débats au sein de l'Église qui avait fini par considérer, au Concile de Latran IV (1215) comme peccamineuses les relations charnelles des fiancés en attente du mariage ${ }^{15}$. En 1590, le tribunal de Cuenca condamna le curé du village d'Altanejos qui avait approuvé publiquement ces relations.

16 L'Inquisition espagnole fit, d'une opinion largement admise dans la population, une proposition hérétique qu'elle châtia avec une mansuétude toute relative, comme nous le verrons plus loin. Il ne s'agissait pas d'éradiquer une pratique, ce que, s'agissant de la prostitution, le Saint-Office aurait eu bien du mal à faire, mais d'inculquer le sens du péché à bon nombre d'hommes et de femmes qui jusqu'alors ne considéraient pas qu'il était aussi grave que cela de laisser libre au cours à leurs pulsions sexuelles. Le but poursuivi n'était donc pas de contraindre les corps, mais tout au plus d'apprendre aux individus à exercer sur eux-mêmes cette contrainte. Au cas où ils n'auraient pas été capables de réaliser cet effort de discipline, et ce point nous semble essentiel, il s'agissait de convaincre ces hommes et ces femmes de s'en remettre au confesseur pour l'absolution de leurs péchés.

17 L'exemple d'une affaire survenue en 1583 dans une taverne de Cuenca est édifiant ${ }^{16}$. Un soir, trois hommes se trouvent réunis dans le mesón de Luisa López : un certain Juan de Cea, identifié comme receptor, c'est-à-dire receveur et, partant, issu d'un groupe social urbain et plutôt cultivé, originaire de la ville de Grenade et de passage à Cuenca, Pedro Rodríguez qui lui est un tisserand du village de Cardenete et l'on ignore l'identité du dernier, un 
montagnard de Carboneras, aux dires des témoins. Lorsque Juan de Cea se lève en disant qu'il va de ce pas visiter le bordel municipal, chacun y va de son commentaire. Dans la conversation qui s'engage, Pedro Rodríguez affirme qu'il n'y a pas péché mortel à aller voir une prostituée. Luisa López lui répond alors : "quien dize tal mire que cada día açotan por la Inquisición ». Sur ce Juan de Cea s'en va et, le lendemain, Luisa López dénonce Pedro Rodríguez. Peu importent les actes, il s'agit simplement de dire que l'on est conscient de la nature du péché commis pour satisfaire, dans ce domaine, les exigences du tribunal de la foi. C'est pourquoi il me semble essentiel de considérer que cette discipline imposée par l'Inquisition vise, en dernier ressort, à renforcer le rôle de la confession dans le dispositif de contrôle idéologique et social post-tridentin.

18 Il en va de même pour toute une série d'affirmations remettant en question l'obligation annuelle de se confesser, ou bien l'efficacité du sacrement de la pénitence administrée à l'issue de la confession, ainsi que, bien sûr, de la répression de l'acte de sollicitation, outrage fait au sacrement de la confession tel que le définissait et l'administrait l'Église catholique.

19 L'examen des sentences prononcées et des peines infligées ${ }^{17}$ retiendra maintenant notre attention.

20 Un premier constat s'impose à la lecture des chiffres : rares sont les instructions débouchant sur ce que l'on appellerait aujourd'hui un non lieu : 1,1 \% des causes dont la sentence est connue. Dans le doute, les inquisiteurs préféraient prononcer une suspension du procès, laissant ainsi planer sur l'accusé l'épée de Damoclès d'une éventuelle réouverture de l'instruction. Tel fut le cas de $37 \%$ des causes étudiées. Ces deux valeurs relatives sont stables tout au long de la période considérée.

21 À l'autre extrémité du spectre des sentences, l'on ne trouve qu'un seul condamné à mort pour entrave à l'action du Saint-Office, et seulement 10 réconciliés, soit 0,5\% de l'ensemble ( 1 blasphémateur, 5 pour propositions dont 1 pour simple fornication et 1 pour erreur sur la hiérarchie des états, 1 pour bigamie, 1 pour outrage à un objet de culte, 


\title{
2 renégats).
}

22 Dans la majorité des cas étudiés (61\%, et ce de façon homogène pendant les quarante années couvertes par cette étude) l'instruction déboucha sur une condamnation, comprenant au moins, l'administration d'une pénitence, assortie parfois, d'amendes et de châtiments infamants.

23 Que retirer de ces chiffres ? Une première constatation : le contraste flagrant entre le nombre d'affaires traitées et le nombre de réconciliations prononcées. De toute évidence, les inquisiteurs ne considérèrent comme hérétiques qu'une part infime des vieux-chrétiens tombés dans leurs filets, même si, dans les faits, ils en traitèrent de nombreux comme tels en leur ordonnant de paraître en public à la cérémonie de l'autodafé.

24 Prenons un exemple ${ }^{18}$. D'après une liste où figurent les noms, les délits et les peines des quarante individus que l'on avait prévu de faire paraître à l'autodafé qui allait se tenir en août 1583 , l'on trouve $^{19}$ :

\author{
9 morisques \\ 1 faux témoignage \\ 7 « revocantes » \\ 1 «luthérien» \\ 1 proposition hérétique \\ 2 sorcellerie \\ 1 blasphème \\ 1 opposant à l'action du Saint-Office \\ 17 « simple fornication »
}

25 Parmi les 22 condamnés pour des délits mineurs (à l'exclusion donc des morisques, du "luthérien », des 7 individus n'ayant pas respecté les termes de leur châtiment et du parjure) aucun ne fut réconcilié. Le détail des châtiments infligés, recueilli dans le tableau $\mathrm{n}^{\circ} 3$ montre une certaine régularité :

celui qui fut condamné pour s'être opposé au SaintOffice ne dut pas abjurer, c'est le seul du groupe tous les autres, c'est-à-dire ceux qui furent condamnés 
pour sorcellerie, propositions, dont la simple fornication et le blasphème durent abjurer

14 des 22 condamnés eurent à subir une peine de bannissement allant de un à deux ans de leur village de résidence assortie, parfois, d'une interdiction de séjour dans la ville de Cuenca

le châtiment corporel (100 coups de fouet) infligé à 8 condamnés est une alternative à l'amende, car aucun des condamnés ne fut astreint aux deux peines. Dans l'un des cas, le châtiment du fouet fut commué en amende eu égard à l'âge du condamné. Les amendes s'échelonnent entre 2000 et 12000 maravédis le bâillon est réservé au blasphémateur du lot la réclusion dans un monastère concerne un seul condamné, identifié comme Français, coupable d'avoir tenu des propos teintés d'hérésie.

L'année 1583 appartient à la période où les tribunaux péninsulaires mirent l'accent sur la répression de la " simple fornication ». Selon la carta acordada de 1574, leur action répressive devait être complétée par une campagne d'instruction à la charge des curés ${ }^{20}$. Jean-Pierre Dedieu, se réfère à l'ouvrage d'un chanoine de Salamanque, Francisco Farfán, paru en 1585 sous le titre éloquent : Tres libros contra el pecado de la simple fornicación, donde se averigua que la torpeza entre solteros es pecado mortal, según ley divina, natural y humana, y se responde a los engaños de los que dicen que no es pecado et affirme à juste titre que l'Église tout entière s'était mobilisée pour convaincre les fidèles du caractère illicite de cette opinion.

27 Deux autres documents relatifs à cette même année 1583 indiquent le détail des sentences prononcées à l'issue de 40 instructions de causes mineures (Tableau $n^{0} 4$ ) et confirment cette priorité donnée à la condamnation de cette proposition spécifique $^{21}$. Sur 28 condamnés pour propositions scandaleuses, 23 le furent pour le délit de « simple fornication ", 4 pour propositions diverses et 1 pour avoir nié la supériorité du célibat sur l'état de mariage. Alors que ces 5 derniers furent condamnés à abjurer et, 
partant, considérés comme hérétiques, ce traitement ne fut pas appliqué de façon systématique aux « fornicateurs » puisque 19 abjurèrent sur 23. Cependant, c'est sur cette population que se sont abattues les peines les plus lourdes et les plus " impressionnantes » telles une amende (8 individus), le fouet (7), le bannissement (6) et le costume d'infamie (4), aucun de ces châtiments n'ayant été infligé aux 5 autres condamnés mentionnés plus haut. L'on relèvera l'importance accordée au blasphème puisque les 4 individus condamnés pour ce délit durent abjurer, puis paraitre en public revêtus d'un bâillon, 2 d'entre eux furent bannis de leur village, 1 seul reçut le fouet et, enfin, un dernier fut envoyé aux galères royales. Parmi les 4 religieux sollicitants, 3 furent mis hors d'état de nuire par une condamnation à la réclusion dans un monastère.

L'examen des sentences prononcées à l'encontre des individus condamnés pour ces délits mineurs montre le souci de l'institution de réserver la plus grande publicité aux opinions et aux conduites qu'elle considère comme prioritaires dans l'entreprise de disciplinement des sujets de la monarchie catholique qu'elle conduit conjointement avec l'Église. À l'inverse, s'agissant des religieux sollicitants, la mise à l'écart des coupables traduit la préoccupation de ne pas nuire à la réputation du clergé. La politique de communication de l'Inquisition est déterminée en fonction du public que l'institution cherche à intimider et à instruire : en 1575, le Conseil Suprême ordonnait aux inquisiteurs de Cuenca de convoquer tous les supérieurs des couvents et des monastères de la ville pour assister, dans la salle d'audience du tribunal, loin du public, à la lecture de la sentence prononcée contre un confesseur indélicat.

29 S'il est assez aisé d'appréhender les moyens que les tribunaux inquisitoriaux mettent en ouvre pour diffuser une information en fonction du public visé, mesurer l'efficacité de cette politique l'est beaucoup moins. La lecture des procès, particulièrement l'étude des déclarations des accusés et des témoins, n'apporte qu'un éclairage partiel sur la question, et sans doute biaisé, puisqu'il ressort de ces documents que les témoins, et parfois l'accusé lui-même, 
sont conscients et informés du caractère délictueux de telle opinion ou conduite. Mais jusqu'à quel point ces individus sont-ils représentatifs de la société de leur temps ? Sans prétendre apporter une réponse définitive à cette question, il me semble intéressant de considérer deux éléments susceptibles d'éclairer ce problème d'un autre jour : le premier est la distribution géographique de la répression inquisitoriale ; quant au second, il s'agit des visites du district de Cuenca.

30 Voyons le premier. En m'intéressant à l'origine géographique des accusés de délits mineurs, j'ai essayé d'évaluer quelle était l'emprise du tribunal de Cuenca sur l'ensemble de son district ${ }^{22}$. Les résultats de cette étude sont présentés dans la cartographie en annexe (Cartes 1, 2 et 3). Si l'on considère que la correspondance entre les villes et les villages existant au $\mathrm{XVI}^{\mathrm{e}}$ siècle et ceux d'aujourd'hui est parfaite, l'on trouve que seulement la moitié (51\%) des villages ont eu un habitant au moins jugé par le Saint-Office au cours des quarante années couvertes par cette étude. Ce rapport est à manier avec précaution étant donné qu'il se peut que certains villages actuels n'existaient pas en tant que tels au $\mathrm{XVI}^{\mathrm{e}}$ siècle ; retenons cependant ce premier constat : l'Inquisition n'était peut-être pas aussi omniprésente sur l'ensemble du territoire que l'on serait tenté de le croire.

31 Si l'on ne tient pas compte, cette fois, du lien entre la topographie ancienne et l'actuelle et que l'on se limite à prendre en considération les 356 lieux qu'il est possible de situer sur une carte, l'on peut tirer un certain nombre d'enseignements quant à la répartition géographique de la répression. J'ai distingué, pour plus de clarté, la répression sporadique (de 1 à 4 cas pour la période de 40 ans, Carte $\mathrm{n}^{\circ} 3$ ) de la répression plus systématique ( 5 cas et plus, Carte $\mathrm{n}^{\mathrm{o}} 2$ ).

32 En toute logique, la ville de Cuenca, siège du tribunal, rassemble, à elle seule, 227 cas, soit près de $9 \%$ du total des affaires instruites. Au-delà de ce premier constat, l'on observe que l'essentiel de la répression systématique touche les villages situés au Sud du district dans la région de la 
Mancha. Parmi les 8 villes citées plus de 35 fois, 6 se trouvent dans cette zone ; San Clemente (125), Huete (117), Belmonte (83), Villaescusa de Haro (38), Villanueva de Alcardete (38) et Santa María del Campo (37). Mais en dehors de ces 6 bourgs qui subissent la répression la plus forte, c'est l'ensemble de cette région qui est plus lourdement frappé que le reste du district. Comme l'indique la carte $\mathrm{n}^{\circ} 2$, la plupart des villages ayant eu plus de 5 habitants jugés par l'Inquisition se trouvent dans cette partie sud du district et dès que l'on s'éloigne de la Mancha vers l'Est ou vers le Nord, cette répression systématique se fait de plus en plus rare et reste limitée aux bourgs les plus peuplés que sont Requena (27), Berlanga (28), Sigüenza (35), Almazán (47) et Molina (75).

33 Examinons maintenant la carte $\mathrm{n}^{\circ} 3$ où est représentée la répression que j'ai appelée sporadique, entre 1 et 4 procès, soit un tous les dix ans au maximum. La répartition de ces villages, relativement homogène dans la totalité du district, présente une concentration légèrement plus élevée au centre et dans la partie nord du district.

34 La superposition des deux cartes montre que pendant ces quarante années, le tribunal de Cuenca a fait sentir sa présence sur la totalité du district soumis à sa juridiction, quoique de façon bien différente selon qu'on se situe au Sud ou au Nord d'une ligne allant de Guadalajara à Utiel et passant par Cuenca. Cette ligne sépare approximativement la Mancha du reste du district inquisitorial. Au-dessus de cette frontière géographique, le tribunal est présent mais de façon très irrégulière. Son activité se concentre sur les bourgs les plus peuplés et, occasionnellement, d'autres lieux, plus isolés et moins peuplés, sont touchés : si la présence du tribunal se fait sentir, c'est d'une façon plus diffuse qu'au Sud du district. En dessous de cette ligne, dans les plaines de la Mancha, cette présence est beaucoup plus forte et constante.

35 Ces différences correspondent bien sûr à l'hétérogénéité géographique du territoire formant le district de Cuenca et aux différences existant entre la plaine de la Mancha, au Sud, fortement peuplée, et les zones plus montagneuses de 
la serranía de Cuenca, au centre, ou de la alcarria, au Nord, plus sauvages et à la moindre densité de population. Mais cela n'explique pas tout. D'autres facteurs doivent être pris en compte.

36 Plus proche et plus peuplée, la Mancha est un terrain d'action plus rentable que les villages isolés des montagnes $\mathrm{du}$ Nord. C'est également une région stratégique, importante zone de communication entre Madrid et la Méditerranée, Valence et son port notamment, sur laquelle il est important d'exercer un contrôle plus strict qu'ailleurs. En outre, la comparaison de ces résultats avec la localisation des foyers de crypto-judaïsme est éloquente. Cette partie du district de Cuenca est celle qui a fait l'objet de la plus forte répression anticonverse quelques années auparavant. Enfin, c'est dans cette même aire géographique que se sont établis de nombreux morisques grenadins déportés à l'issue de la guerre des Alpujarras ${ }^{23}$. Il est donc probable que soit déjà en place un réseau de contrôle de la population susceptible d'être mobilisé contre les nouvelles cibles du tribunal inquisitorial. Tout cela concourt à faire de cette partie de la Mancha, une zone particulièrement importante à surveiller, alors que la partie septentrionale du district, mis à part les villes dont il a été fait mention plus haut, fait l'objet d'une surveillance nettement plus lâche.

37 Le second élément à prendre en compte est celui des visites. Elles sont peu nombreuses et mal connues dans la seconde moitié du $\mathrm{XVI}^{\mathrm{e}}$, mais pour ce qui est du tribunal de Cuenca, elles me semblent particulièrement intéressantes car elles concernent cette zone moins surveillée qu'est le Nord du district. Nous examinerons ici le compte rendu de deux visites : la première date de 1569 et se déroula à Almazán et dans les villages alentour ${ }^{24}$; la seconde eut lieu en 1583 à Sigüenza et dans son évêchée ${ }^{25}$.

38 Le document mentionnant la visite de 1569 fait apparaitre, sans plus de détails pour ce qui est des délits mineurs qui nous intéressent ici, la liste de 26 individus à qui l'Inquisiteur imposa des peines spirituelles. Le compte rendu de la visite de 1583 est plus détaillé. Il apparaît que plusieurs dénonciations ou présentations spontanées 
pendant le délai de grâce ne donnèrent lieu à aucune autre procédure qu'une simple admonestation verbale assortie de l'obligation de se confesser. Ainsi à Berlanga, un certain Juan de Soria qui s'était présenté de son propre chef en s'accusant d'avoir tenu des propos niant le caractère peccamineux de la « simple fornication » en fut quitte pour une confession. Le cas n'est pas isolé, bien que d'autres individus aient fait l'objet d'une instruction pour les mêmes délits sans que l'on dispose d'éléments permettant d'expliquer pourquoi la même cause ne produit pas toujours les mêmes effets.

39 Loin du siège du tribunal, et répétons-le, dans ce type d'affaires où il n'est question que de délits mineurs, l'Inquisiteur semble davantage enclin à la clémence en renvoyant de nombreux coupables devant le confesseur sans passer par l'étape de la procédure judiciaire.

40 Ces quelques constats me conduisent à reconsidérer l'appréhension de ces phénomènes en fonction des rapports entre Inquisition et confession. Des rapports dont l'histoire des premiers temps de l'Inquisition espagnole donne peut-être une vision trop antagoniste. Lors de l'établissement des tribunaux péninsulaires, cette question s'était trouvée au coeur du conflit qui opposa le Saint-Siège et la nouvelle inquisition espagnole : la Bulle de Sixte IV de 1482 qui donne à l'ordinaire ou à ses délégués, ainsi qu'aux inquisiteurs, le pouvoir d'absoudre les judéo-convers se présentant spontanément pour confesser leurs erreurs, et qui étend cette absolution du for intérieur à l'externe, annulant ainsi toute procédure inquisitoriale, nous rappelle deux choses : en premier lieu, que confession et inquisition poursuivent avec des moyens différents le même objectif de réintégration / réconciliation du pécheur / hérétique à la communauté chrétienne ; ensuite, qu'il existe bien la possibilité d'effacer par l'absolution le crime d'hérésie. L'on sait que Sixte IV finit par faire machine arrière sur cette question et que, dans les affaires de crypto-judaïsme, l'Inquisition conserva son monopole : la pratique de l'absolution en for intérieur fut définitivement écartée au profit de la procédure inquisitoriale. 
41 Les choses sont moins claires en ce qui concerne les délits secondaires qui nous ont intéressé ici. Sans aller jusqu'à analyser l'activité de l'Inquisition espagnole au prisme des thèses d'Adriano Prosperi ${ }^{26}$, élaborées à partir de l'étude d'une Inquisition italienne agissant dans un contexte politique, religieux et social profondément différent de celui de la péninsule ibérique, il me semble fécond d'en retenir certains éléments. Dans les affaires dites mineures, et notamment la condamnation des propositions diverses, des pratiques magiques, de la sollicitation et de la bigamie, l'action du Saint-Office espagnol se décline selon deux registres complémentaires, répression et persuasion. L'inquisiteur, infligeant des châtiments exemplaires dans le but d'impressionner les fidèles mais n'hésitant pas non plus à faire preuve de clémence en se contentant de prononcer des pénitences spirituelles, contribue à sa manière à renforcer la place de la confession dans le dispositif de disciplinement social à l'œuvre dans la seconde moitié du $\mathrm{XVI}^{\mathrm{e}}$ siècle.

42 En quelque sorte, l'Inquisition espagnole a un double visage. Celui des premiers temps de l'existence du tribunal où ce dernier s'est employé à éliminer, avec la brutalité que l'on sait, un nombre effarant de judéo-convers considérés, à tort ou à raison, comme judaïsants. Détentrice de la mémoire de cette entreprise, l'Inquisition a été ensuite l'instrument de la pérennisation de l'exclusion dont furent victimes la plupart de leurs descendants au nom de la pureté de sang. Son autre visage est celui d'une institution au service d'un projet de disciplinement religieux, social et politique assumé par la monarchie et par une partie des autorités ecclésiastiques dont je n'ai fait ici qu'aborder quelques aspects. 

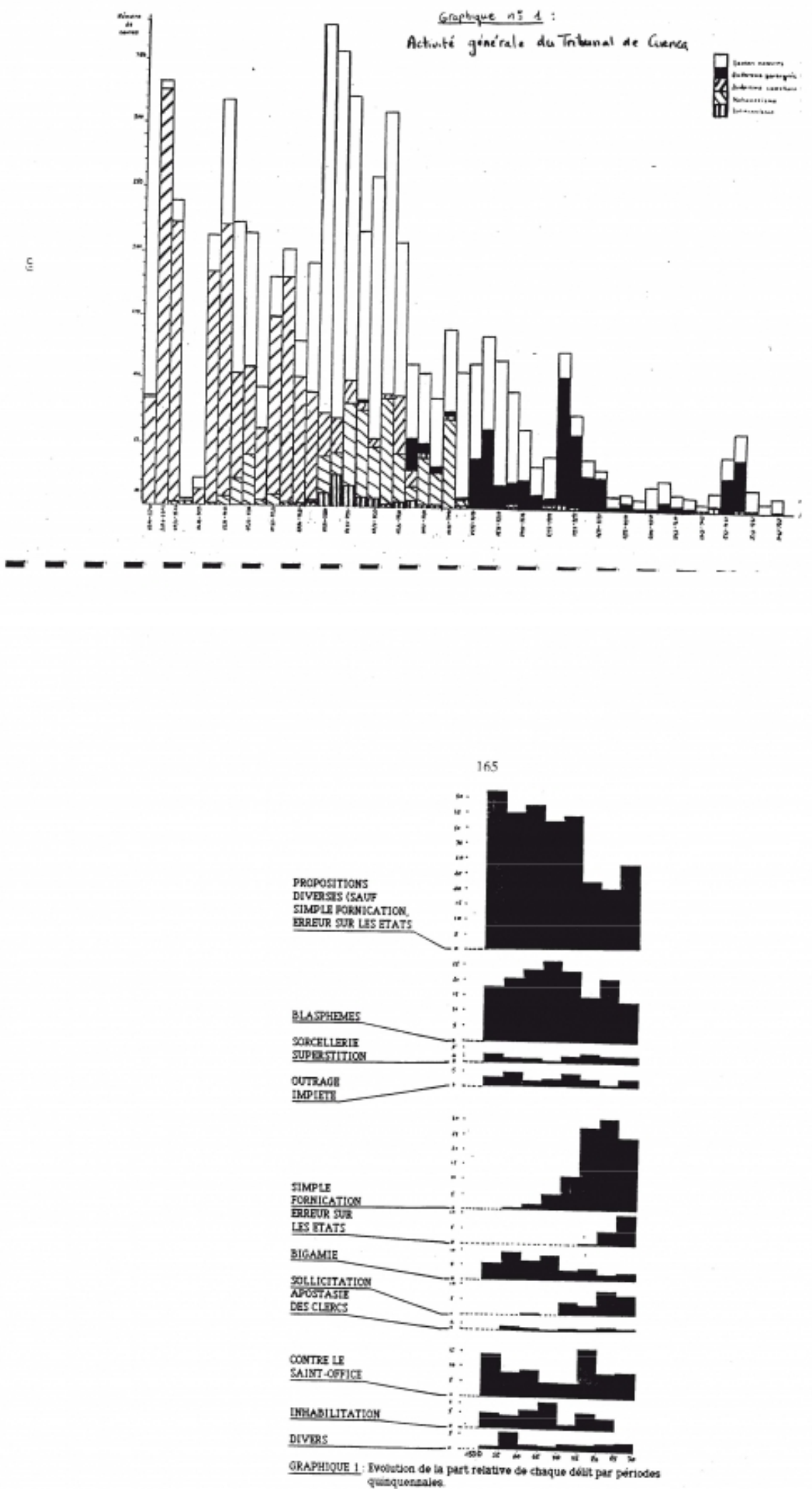


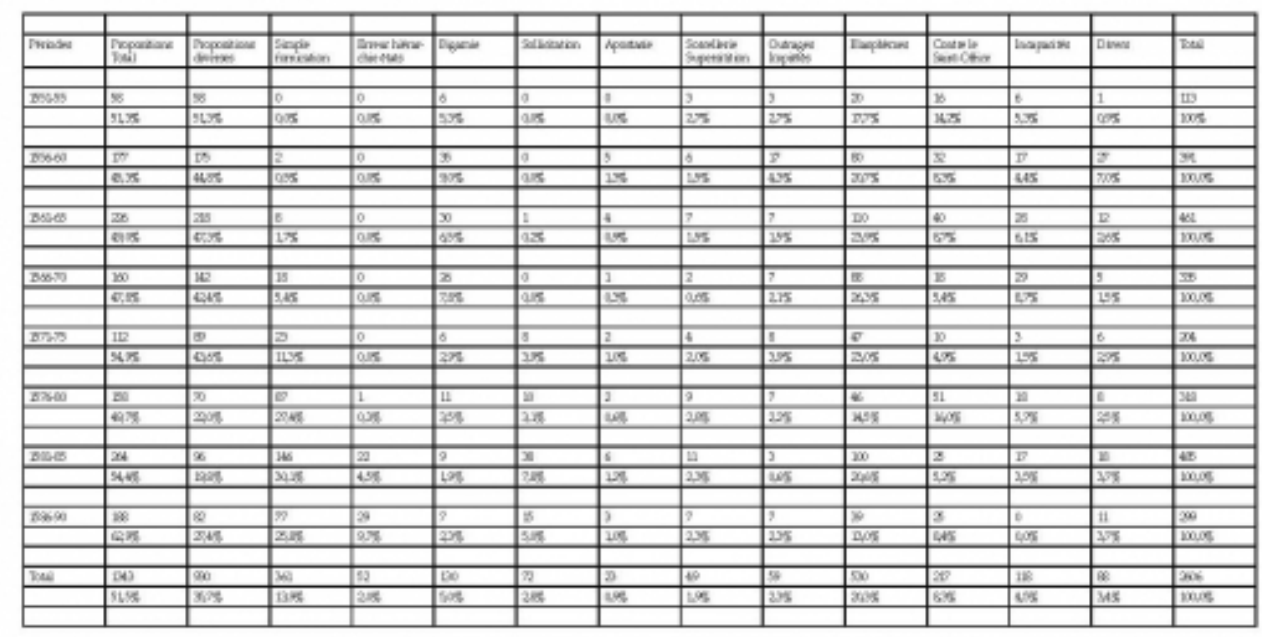

\begin{tabular}{|c|c|c|c|c|c|c|c|c|}
\hline Peniodes & Albodution & Penitence & Rtocoscilitations & $\begin{array}{l}\text { Condamnations a } \\
\text { moot }\end{array}$ & Suspension & 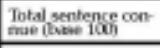 & Sentence incosenve & Total \\
\hline \multirow[t]{2}{*}{1551.55} & 4 & 6 & 1 & 0 & 25 & 96 & 17 & 113 \\
\hline & 425 & $x, 85$ & $1,0 \%$ & $0.0 \%$ & 2605 & & & \\
\hline \multirow{3}{*}{155660} & & & & & & & & \\
\hline & 2 & 165 & 0 & 1 & 131 & 299 & $x$ & 991 \\
\hline & $0.3 \%$ & 55,236 & 0.05 & a.9\% & 43.95 & & & \\
\hline \multirow{3}{*}{$1561-65$} & & & & & & & & \\
\hline & 1 & 260 & 1 & 0 & 154 & 418 & 4 & 461 \\
\hline & 0,25 & $2,7 \%$ & 0.25 & $200 \%$ & 36,95 & & & \\
\hline \multirow[t]{2}{*}{136670} & 1 & 173 & 0 & 0 & 106 & 282 & 53 & 585 \\
\hline & 0.48 & $61.3 \%$ & 0.5 & 0,005 & $35,5 \%$ & & & \\
\hline & & & & & & & & \\
\hline \multirow[t]{2}{*}{$1571-75$} & 2 & 106 & 4 & 0 & 68 & 19 & 25 & 204 \\
\hline & 1,155 & $58,7 \%$ & 223 & $0,0 \%$ & 38,05 & & & \\
\hline \multirow{2}{*}{$1576-50$} & 4 & 160 & 2 & 0 & 84 & 258 & ส & 515 \\
\hline & 1,68 & $6.7 \%$ & $0 \mathrm{sen}$ & $00 \%$ & 30,96 & & & \\
\hline & & & & & & & & \\
\hline \multirow[t]{2}{*}{$1551-85$} & 3 & 221 & 1 & 0 & 129 & 354 & 131 & 455 \\
\hline & 0,856 & $2,6 \%$ & $0,3 \times$ & $0,0 \%$ & 36,45 & & & \\
\hline & & & & & & & & \\
\hline \multirow[t]{2}{*}{1586,90} & 6 & 95 & 1 & 0 & $\oplus$ & 174 & 125 & 89 \\
\hline & $3,4 \%$ & $56.5 \%$ & $0.0 \%$ & $00 \%$ & $59, \%$ & & & \\
\hline & & & & & & & & \\
\hline \multirow[t]{2}{*}{ Iotal } & 23 & 1259 & 10 & 1 & 766 & 2057 & 549 & 2006 \\
\hline & 1,15 & $61,1 \%$ & $0,5 \mathrm{~m}$ & 0,05 & $37,2 \pi$ & & & \\
\hline
\end{tabular}

\begin{tabular}{|c|c|c|c|c|c|c|c|c|c|}
\hline & & & & & & & & & \\
\hline Condemet & Delit & Ablo de lesi & Yerer & Batliben & Fouet & Amente & Relchanion & Burraimement & Gallinem \\
\hline Ana de Sind oval & Sementhrie & oui & & & & & & coui & \\
\hline Mangdalena de is Crus & Soncetlenie & out & ori & & & & & oui & \\
\hline Antide Goreales & 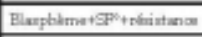 & ori & & & oui & & & \begin{tabular}{|l|l} 
oui \\
\end{tabular} & оші \\
\hline Pasood Saruso & Simpose Ferricution & oú & & & oui & & & 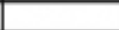 & \\
\hline Francimo Ahrasuz & Simple Forricution & oui & & & & & & & \\
\hline MartnGarcia & Simple Estriction & ou & & & ouil & & & oui & \\
\hline Fudro Peesture & Simple Forrication & oui & & & oui & & & oui & \\
\hline MartinGaxon & Simpose Fomication & out & & & wit & & & & \\
\hline Parnolomide Proreta & Simpin Frerication & oui & & & oui & & & cuil $>$ of & \\
\hline Femando Alonto & Simple Fomrichtion & out & & & oui & & & oui & \\
\hline Dheso de Yrienta & Imple Fyrrication & ou & & & [oui "s menende] & oui & & & \\
\hline Fodro Sancive & Simpie Fertiovion & oú & & & & & & oui & \\
\hline Fedro Bodriguese & Simple Forrication & oxi & & & & oni & & -5 & \\
\hline Fudro Ruis & Simple Feriction & ou & & & & oui & & \begin{tabular}{|c|c|} 
sui \\
\end{tabular} & \\
\hline Futo Puis & Simple Forrication & oui & oui & & & onit & & & \\
\hline Franciues de Seroxdo & Simple Forriction & कां & & & & oxi & & oui & \\
\hline Alonos Nwwanto & Simple Forrication & oui & oui & & & & & sui & \\
\hline Fudro Martine & Simple Fureriotion & ou & oui & & & & & & \\
\hline Ans Mirdiana & Simple Forrication & out & oui & & & & & soui & \\
\hline Fuan Hocelinons & Contre he Saint. Othos & & & & & & & sui & \\
\hline Alsoso Sincies & Bliopbeame & ou & & oul & & & & oui & \\
\hline Entwer de la Grarias & Froupowion brestique & oui & & & & & oui & & \\
\hline Donal:22 & & 21 & 5 & & 7 & 5 & 1 & 14 & 1 \\
\hline
\end{tabular}




\begin{tabular}{|c|c|c|c|c|c|c|c|c|c|}
\hline $\begin{array}{l}\text { Delits / } \\
\text { Sentences }\end{array}$ & $\begin{array}{l}\text { Total indi- } \\
\text { vidus }\end{array}$ & $\begin{array}{l}\text { Abjuration } \\
\text { ade Lavi. }\end{array}$ & Amende & $\begin{array}{l}\text { Costume } \\
\text { d'infamie }\end{array}$ & Baillon & Fouet & $\begin{array}{l}\text { Bsmnisse- } \\
\text { ment }\end{array}$ & Galares & Rédustion \\
\hline $\begin{array}{l}\text { Total proposi- } \\
\text { tions }\end{array}$ & 28 & 24 & 9 & 4 & & 7 & 6 & & 1 \\
\hline & & & & & & & & & \\
\hline Proposite div & 4 & 4 & 1 & & & & & & 1 \\
\hline & & & & & & & & & \\
\hline Simple fornic. & 23 & 19 & $B$ & 4 & 0 & 7 & 6 & & \\
\hline & & & & & & & & & \\
\hline Emeur/ witatsi & 1 & 1 & & & & & & & \\
\hline & & & & & & & & & \\
\hline Blssphème & 4 & 4 & 1 & & 4 & 1 & 2 & 1 & \\
\hline & & & & & & & & & \\
\hline Sollicitation & 4 & 2 & & & & & & & 3 \\
\hline & & & & & & & & & \\
\hline Sorcellerie & 1 & 1 & & & & & 1 & & \\
\hline & & & & & & & & & \\
\hline $\begin{array}{l}\text { Résistanco } \\
\text { au } 50\end{array}$ & 2 & & 1 & & & & & & \\
\hline & & & & & & & & & \\
\hline Inhabilitation & 1 & & 1 & & & & & & \\
\hline & & & & & & & & & \\
\hline Total & 40 & 31 & 12 & 4 & 4 & $B$ & 9 & 1 & 4 \\
\hline
\end{tabular}




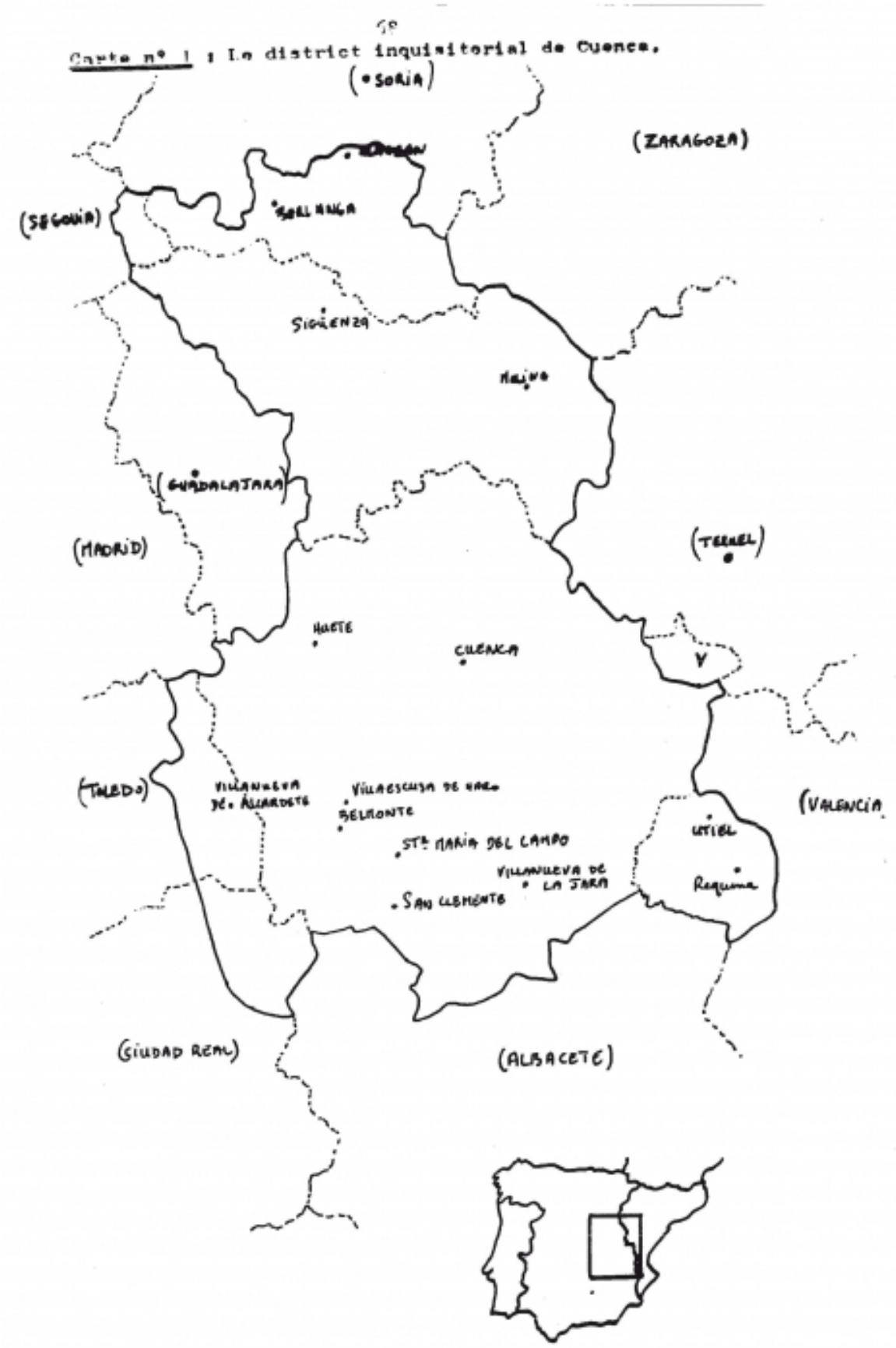




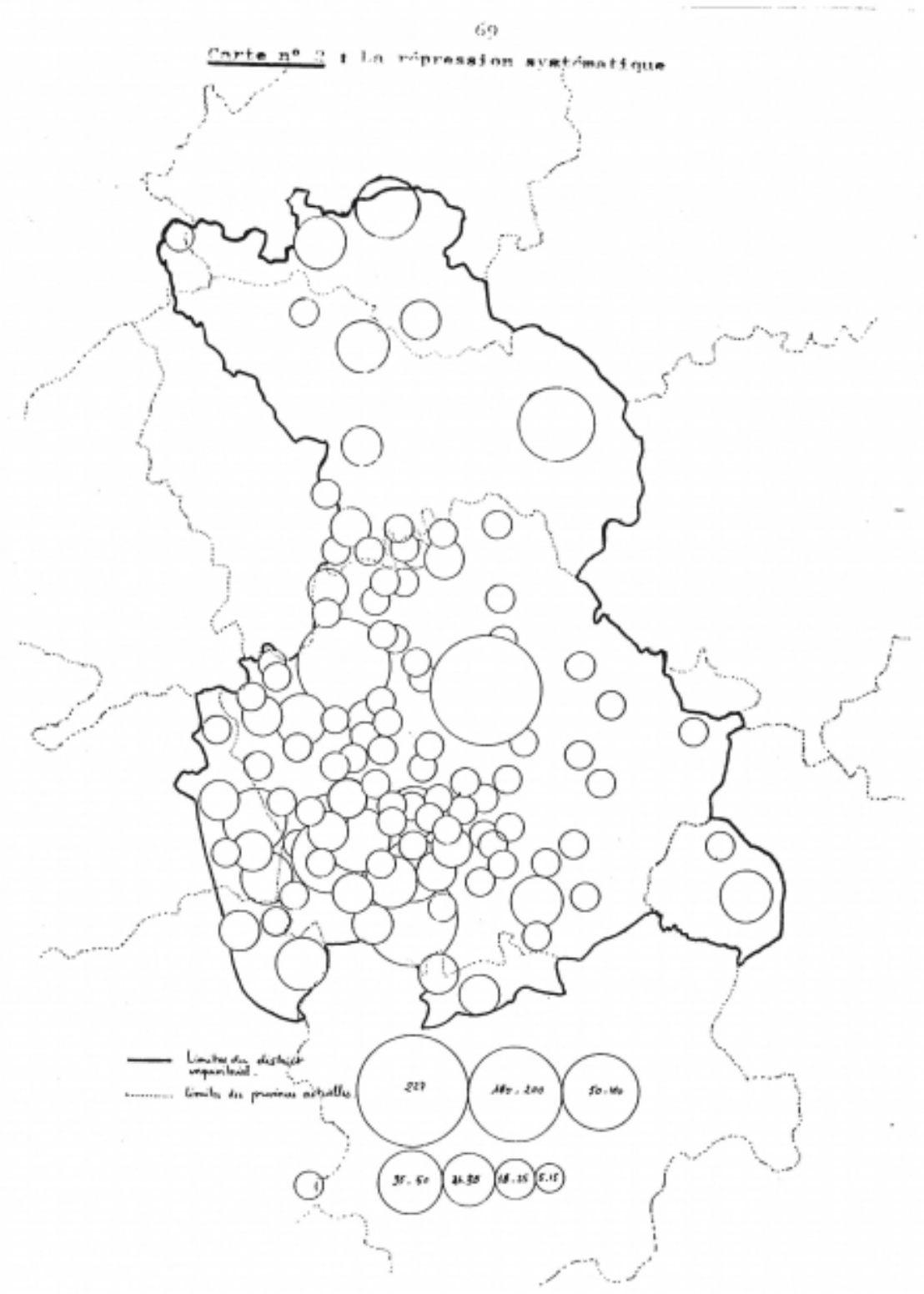




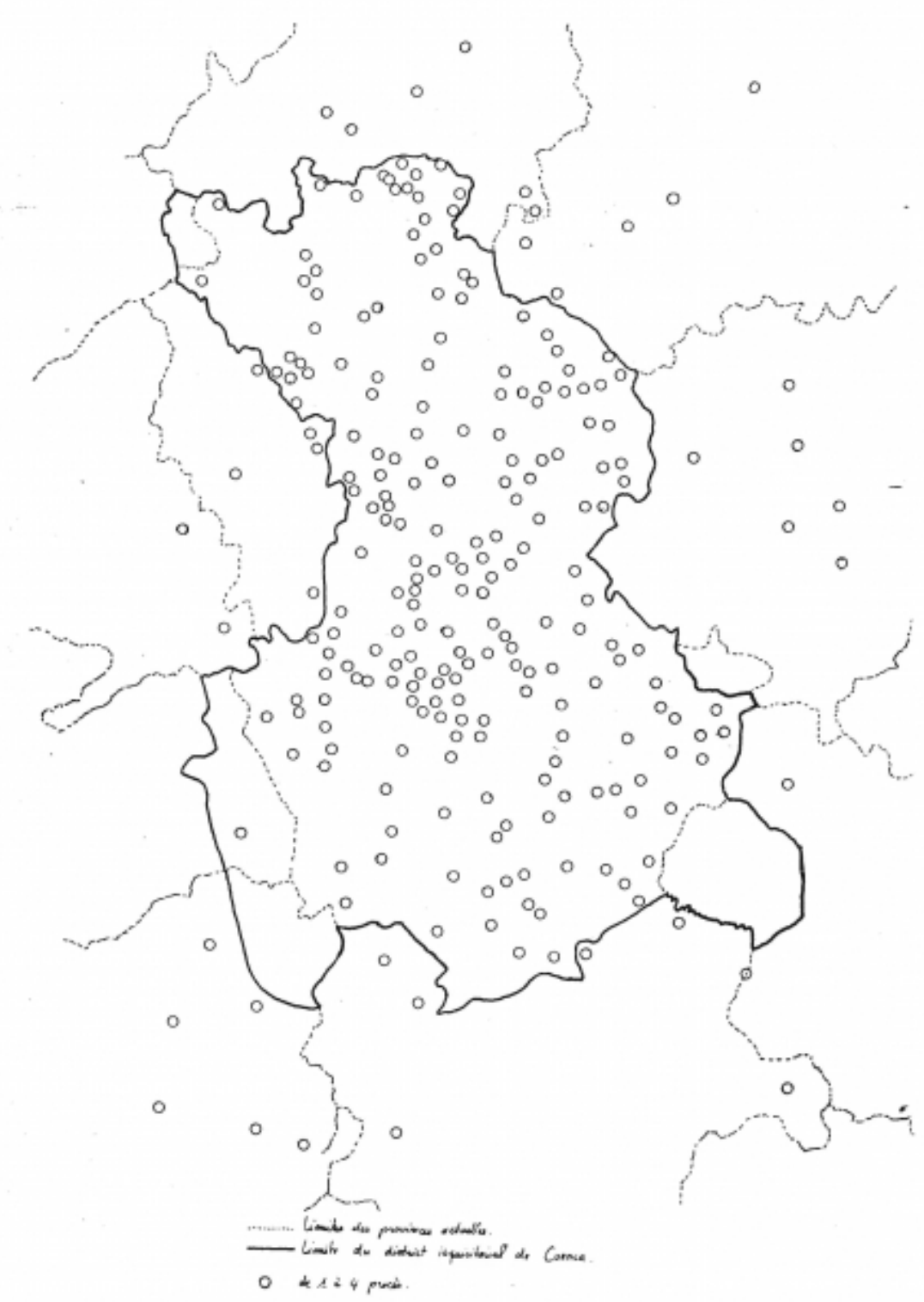

\section{Notes}

1. Jean-Pierre DEDIEU, L'administration de la foi. L'Inquisition de Tolède (XVI ${ }^{e}-X V I I I^{e}$ siècles), 2ème édition, Bibliothèque de la Casa de Velázquez $\mathrm{n}^{\circ}$ 7, Madrid, 1992.

2. André FERNANDEZ, Inquisition et répression sexuelle dans la Couronne d'Aragon (1565-1700), Thèse doctorale dirigée par Raphaël Carrasco, Université de Montpellier, 1998.

3. L'on se rapportera aux travaux de l'historiographie italienne, plus particulièrement à ceux réunis par Paolo PRODI, Disciplina dell'anima, disciplina del corpo e disciplina della società tra medioevo ed età moderna, Bolonia, 1994, ainsi qu'aux réflexions formulées par Federico PALOMO, «Disciplina christiana. Apuntes historiográficos en torno a la disciplina y el disciplinamiento social como categorías de la historia religiosa de la alta edad moderna », p. 119-136, Cuadernos de Historia Moderna, $\mathrm{n}^{\circ}$ 18, Universidad Complutense, Madrid, 1997. 
4. Pascal GANDOULPHE, « Mentalités populaires et christianisation en Nouvelle Castille. Le tribunal de l'Inquisition de Cuenca et les vieuxchrétiens, (1550 - 1590) », Solidarités et sociabilités en Espagne (XVI $X X^{e}$ siècles), (Besançon, décembre 1988), p. 141 - 165, Annales Littéraires de l’Université de Besançon, Les Belles Lettres, 1990.

5. Voir tableau 1.

6. L'on entendait par « simple fornication » l'accouplement volontaire d'un homme et d'une femme libres de tout lien matrimonial, ce qui excluait le viol, l'adultère, l'inceste, la sodomie et la bestialité, qui constituaient la fornication qualifiée.

7. Il en va de même pour celui de Tolède étudié par Jean-Pierre Dedieu.

8. Pendant la décennie 1575-1585, la condamnation de la « simple fornication » représenta plus du quart de l'activité du tribunal de Cuenca et plus du tiers des seules causes « mineures ».

9. ADC Inq Leg 3264681.

10. ADC Inq Leg 2934131.

11. ADC Inq Leg 2934137.

12. Jean-Pierre DEDIEU, « Les causes de foi de l'Inquisition de Tolède (1483-1820). Essai statistique », Mélanges de la Casa de Velázquez, XV, 1978, p. 261-294.

13. ADC Inq Leg 2792544.

14. ADC Inq Leg 2172652.

15. Jean GAUDEMET, Le mariage en Occident, Les Éditions du Cerf, Paris, 1987.

16. ADC Inq. Leg 292, $\mathrm{n}^{\circ} 4138$.

17. Voir tableau $\mathrm{n}^{\circ} 2$.

18. Voir tableau $\mathrm{n}^{\circ} 3$.

19. ADC Inq. Leg $2545, n^{\circ} 64$.

20. Jean-Pierre DEDIEU, L'administration de la foi. L'Inquisition de Tolède (XVI ${ }^{e}-X V I I I^{e}$ siècles), ze édition, Bibliothèque de la Casa de Velázquez $\mathrm{n}^{\circ}$ 7, Madrid, 1992, p. 279 et 299-302.

21. ADC Inq. Lib 352 et AHN Inq. Leg 1931.

22. La méthodologie de ce travail est la suivante : les 2606 causes mineures de la période étudiée (1551-1590) font mention du lieu de résidence des accusés. L'on trouve un total de 414 lieux, dont 41 sont extérieurs au district de Cuenca et 17 n'ont pu être localisés avec certitude. Les 356 villes et villages identifiés ont été rapprochés des 687 lieux existant aujourd'hui sur le territoire correspondant à l'ancien district inquisitorial de Cuenca. Ce rapprochement a donné lieu à l'élaboration de la cartographie présentée en annexe. 
23. Mercedes GARCÍA ARENAL, Inquisición y moriscos. Los procesos del tribunal de Cuenca, Madrid, Siglo XXI, 1983.

24. ADC Inq. $\operatorname{Leg} 751, \mathrm{n}^{\circ} 6$.

25. ADC Inq. Leg $751, \mathrm{n}^{\circ} 10$.

26. Voir en particulier : Adriano PROSPERI, « El inquisidor como confesor », p. 63-85, Studia Histórica, Historia Moderna, vol. XIII, 1995, Ediciones Universidad de Salamanca, Asociación Española de Historia Moderna.

\section{Auteur}

\section{Pascal Gandoulphe}

\section{Université d'Aix-Marseille I}

(C) Presses universitaires de la Méditerranée, 2006

Conditions d'utilisation : http://www.openedition.org.lama.univamu.fr/6540

\section{Référence électronique du chapitre}

GANDOULPHE, Pascal. Les causes mineures du tribunal de Cuenca dans la deuxième moitié du XVI $I^{e}$ siècle In : L'Inquisition espagnole et ses réformes au $X V I^{e}$ siècle [en ligne]. Montpellier : Presses universitaires de la Méditerranée, 2006 (généré le 23 mai 2017). Disponible sur Internet : <http://books.openedition.org.lama.univamu.fr/pulm/712>. ISBN : 9782367810935. DOI : 10.4000/books.pulm.712.

\section{Référence électronique du livre}

BARBAZZA, Marie-Catherine (dir.). L'Inquisition espagnole et ses réformes au $X V I^{e}$ siècle. Nouvelle édition [en ligne]. Montpellier : Presses universitaires de la Méditerranée, 2006 (généré le 23 mai 2017). Disponible sur Internet : <http://books.openedition.org.lama.univamu.fr/pulm/689>. ISBN : 9782367810935. DOI : 10.4000/books.pulm.689.

Compatible avec Zotero 